\title{
Optimization of the Intelligent Asset Management System Based on WSN and RFID Technology
}

\author{
Yunyue Chen ${ }^{1}$ and Guanggui Chen $\mathbb{D}^{2}$ \\ ${ }^{1}$ Stern School of Business, New York, 10001 NY, USA \\ ${ }^{2}$ Yangzhou University Yangzhou College of Science and Technology, Jiangsu Yangzhou 225001, China \\ Correspondence should be addressed to Guanggui Chen; 101293@yzpc.edu.cn
}

Received 15 November 2021; Revised 8 January 2022; Accepted 17 January 2022; Published 4 February 2022

Academic Editor: Chih-Cheng Chen

Copyright (c) 2022 Yunyue Chen and Guanggui Chen. This is an open access article distributed under the Creative Commons Attribution License, which permits unrestricted use, distribution, and reproduction in any medium, provided the original work is properly cited.

\begin{abstract}
With the development of Internet of Things (IoT) technology, especially the promotion of perception layer radio frequency identification (RFID) technology and wireless sensor network (WSN), a new way of thinking is provided for asset management. Applying both to the management technology of assets is an effective way to achieve intelligent asset management. This paper proposes an intelligent network applied to an asset management system based on WSN and RFID technologies. The whole intelligent asset management is divided into four layers according to the functional structure. From the top layer downward, the layers are the management and dispatch center, communication network, intelligent gateway, and data collection layer in order. Meanwhile, the functions and hardware composition of each layer are described. The data acquisition butcher is the sensing terminal of the whole intelligent asset management network. Smart nodes are its key devices to collect RFID data and sensing data to realize the intelligent sensing capability of the assets. The paper uses the MSP430 control chip, MFRC522 RF read/write chip, CC2425 wireless communication chip, temperature sensor, data memory, and voltage regulator chip to design the smart nodes in the system and details the working principle of the smart nodes and the networking process of the system. The newly designed intelligent asset management network can be deployed independently in asset management or can be integrated into the technical design of existing asset management systems. The management scheduling also collects and manages the RFID and sensing information of the area through the data collection layer, which improves the intelligent construction of the assets.
\end{abstract}

\section{Introduction}

The corresponding work involved in asset management is tedious, and the process is relatively fixed. It has to involve all kinds of work-related to equipment storage, equipment inquiry, equipment monitoring, equipment maintenance, and equipment change and asset loss analysis [1]. In traditional asset management, a lot of daily management work is done in the traditional manual way, such as regular inventory check of fixed assets, registering the relevant asset and equipment data collected with paper materials, and then entering these data into the computer memory file after manual collation. Fixed assets used in enterprises are characterized by a wide range of types, high values, and a large number of people handling them, a long life cycle, and a wide distribution of locations [2]. The traditional way of management often leads to problems such as incomplete registration information, data not being updated in time, inconvenient information inquiry, and difficulties in integrating information in isolation. By deploying and controlling the various base stations and locators in the area, it is possible to know the asset location and distribution of a certain indoor and outdoor attached asset card in time and record its historical location information [3]. The administrator can query the real-time location of the specified asset in real-time and mark it on the electronic map of the system. The distribution of assets in each depository can be inquired in real-time, and the detailed asset 
information of a certain distribution can be viewed [4]. It can query and replay the historical movement tracks of assets and historical data reports.

This paper carries out research work such as understanding and analyzing the fixed asset and equipment management technology existing in enterprises, using advanced object-oriented technical means to analyze and model the needs of asset management, applying RFID technology to track and locate important equipment, and using smartphones as tools to manage asset information in a timely and convenient manner. Radiofrequency identification technology (RFID) has gradually become a hot research area of concern for current information technology, and a variety of various applications based on this technology are emerging [5]. Radiofrequency identification technology (RFID) works in a very different way from the traditional way, and it uses a noncontact way to automatically acquire and identify the required data. Since RFID technology is not limited by physical location and can communicate with multiple objects at the same time, it has been widely promoted in various applications related to location. Asset management using various technologies is well organized to achieve information and standardized management of assets [6]. The asset management system mainly has functions such as asset registration, data inquiry, change management, asset review, asset inventory, asset statistics, and report printing. The key issues in the development of this system are the setting of network security access rights, diversity of data query, performance optimization of the system, QR code generation and report printing, etc. This paper adopts the scheme of enterprise fixed asset management system with WSN and RFID, taking into full consideration to reduce manual work intensity and improve the management level and efficiency [7]. The choice of WSN and RFID technology program, using its technical advantages of real-time positioning monitoring of valuable fixed assets, can effectively prevent the loss of valuable assets and damage to the enterprise and improve the security of assets and equipment and efficiency of use.

The separation of system operation and maintenance personnel from basic operation and maintenance personnel reduces the cultural and operational requirements of basic operation and maintenance personnel and reduces the personnel requirements for daily operation and maintenance of the system, thereby saving the cost of high-end operation and maintenance manpower. This paper proposes to apply the wireless sensor network and RFID fusion technology to the intelligent asset management system and design the fusion wireless sensor network node and RFID electronic label status monitoring, the main contents of this paper and the related chapters are arranged as follows [8]. The first chapter analyzes the background of this paper and the practical significance of the research in detail and introduces the research work of the paper. The second chapter analyzes the research status of various intelligent asset management systems and condition monitoring devices at home and abroad, explains the advantages of integrating RFID technology with WSN technology, summarizes the advantages and disadvantages of existing research results, and puts forward the research direction and objectives of this paper. Chapter 3 analyzes the intelligent asset management system based on WSN and RFID technology. This paper proposes a new intelligent asset management network, which mainly consists of a condition monitoring IED fusing WSN nodes and RFID tags and a master IED fusing aggregation nodes and readers, and focuses on analyzing the data communication mode between the condition monitoring IED and the master IED. An RFID technology prioritization strategy and RFID and ZigBee communication technology switching algorithm are designed for the intelligent asset management network. Chapter 4 analyzes the research of this paper, tests the intelligent asset management network model and system constructed in this paper, and summarizes the test results. Chapter 5 concludes and outlooks this paper, summarizes the work and research results done in this paper, analyzes the shortcomings that still exist in the system design and implementation, and proposes further research improvement solutions for these shortcomings.

\section{Related Work}

Asset management is an indispensable part of business management, and all companies are paying more attention to equipment assets. A variety of advanced technologies are used to continuously improve the management of assets and the efficiency of use, domestic, and foreign enterprises to reduce the rate of idleness of assets, especially the use of valuable assets, to avoid various losses [9]. Kinnunen et al. used WSN nodes and reader fusion for security monitoring of fixed assets. WSN nodes are responsible for collecting environmental parameters such as temperature, humidity, and pressure of the warehouse where fixed assets are stored and establishing a transmission network to transfer this sensory information to the host computer system simultaneously with the fixed asset information read by RFID technology, thus achieving a real-time mastery of fixed asset warehouse information. In the WSN node and reader fusion network, the reader can join the WSN network; however, RFID tags are still only able to communicate with the reader through RFID technology, which limits the communication range [10]. Andreacchio et al. proposed WSN node and RFID electronic tag fusion for monitoring fixed assets, the information stored in the electronic tag is also regarded as a kind of sensing data, and Zigbee star topology network is used to transmit the sensing data of WSN node and RFID tag information. It is proved through experiments that only $2.44 \mathrm{~W}-2.5 \mathrm{~W}$ more power consumption is consumed by embedding RFID tags into fixed assets. In the network of WSN nodes fused with electronic tags, the data transmission mainly relies on the WSN network, but the power consumption of the WSN network to transmit data is higher [11]. Çeken et al. used RFID tag and sensor fusion so that the RFID electronic tag can sense environmental information; however, the fused RFID tag sensor is only able to communicate with the reader single-hop through RFID technology and cannot join the WSN network, and the communication distance is short [12].

Barcode-based asset management systems are generally used to manage assets in physical form, characterizing an 
asset with a barcode tag to achieve asset management. However, barcodes are not as efficient as RFID technology because they are prone to breakage and can only be read one by one [13]. Chen et al. proposed the structure of WSN and RFID convergence, which includes distribution streamlined sensor-reader structure, hybrid network structure, and intelligent active tag network structure, and analyzed the problems of technology faced by these three systems structures [14]. Chen et al. extended the architecture design of the fusion network and improved the network of intelligent nodes effectively to achieve intelligent management of the location management system of the warehouse and adopted an adaptive system structure used to improve the efficiency and reliability of the inventory goods, and this system has the characteristics of flexibility and stability [15]. Lee et al. fused sensor nodes and RFID tags and divided them into two types of tags, active, and passive and solved the synergistic working mechanism of the two as a key problem [16]. The effective fusion of WSN and RFID technologies can fully solve the existing deficiencies of the existing research, and the wireless RFID devices can work in harsh environments, have a small size, are resistant to interference, have strong penetration, can quickly identify moving objects, and can identify multiple tags simultaneously [17].

The above research shows that RFID and WSN technologies have been widely used in various fields, but the application of these two technologies in the coal industry has just begun, and the application prospects are broad [18]. The RFID system integrated with WSN has the advantage of wireless communication. Compared with wired communication, it is convenient to transmit and process key data and save valuable time. Portable RFID readers can further speed up data collection. From the relevant literature at home and abroad, all the above applications are relatively single, and only two technologies are used separately. The research work of RFID and WSN fusion technology has not fully considered the interference of RFID to ZigBee wireless sensor networks, RFID anticollision algorithms, optimized RFID positioning and tracking technology for intelligent asset management environment. This project will further study the key theories of RFID and WSN fusion technology, establish the positioning model, and play an important role in complementing the positioning algorithm for intelligent asset management. Due to the need for long-distance reading, asset management usually uses UHF RFID technology [19]. This thesis analyzes and studies the architecture composition and function module of RFID technology and studies the interface call and functional interaction between the reader and the two terminals of client and handheld. At the same time, since assets are scattered inside and outside the enterprise, the enterprise does not want the data in the RFID tags attached or installed on the assets not to be known by unauthorized users [20].

\subsection{Multipath Routing Method for Video Streams in Wireless Multimedia Sensor Networks Based on Optimized Ant Colony Algorithm}

2.1.1. General Analysis of the Intelligent Asset Management System. Through the functional and business logic analysis of the asset management system, a deep requirement analy- sis of the system designed and implemented in this thesis is conducted. The four main functional modules of this system are summarized to include system management function, asset management function, asset query function, and report management function. The user rights are divided into the following types: system administrator, departmental asset manager, and general user. The system administrator has all the system privileges and can perform the following four functional operations: system management, asset management, asset inquiry, and report management. The departmental asset manager has three functional rights: asset management, asset inquiry, and report management. Ordinary users have only two basic functional privileges: asset inquiry and report management.

We set the QR code to consist of a UUID (universal unique identification code) and an information part, whose functions correspond as shown in Table 1.

Smart tag is a fusion of RFID tag and wireless sensor node together. Its main structure is divided into a tag module, a processor module, sensor module, wireless communication module, storage circuit, power supply, voltage regulator circuit, etc. The tag is not only able to communicate with RFID readers through tag antenna but also able to communicate with each other through node antenna and other nodes. Smart tags and radio frequency read-write devices by RFID standards for data transmission between the smart tags are by the WSN protocol networking communication. The data forwarded between smart tags include unique electronic code information and monitored environmental dynamic information.

The monitoring and scheduling center is the top department of the whole monitoring network, where the monitoring and display platform can query and monitor all RFID and sensing data in the current network; the linkage response mechanism can quickly execute the order to eliminate faults. The communication network in terms of hardware facilities is mainly for signal towers and related communication devices in the network, and its direct purpose is to transmit the data gathered by the intelligent gateway to the monitoring and scheduling center safely and reliably, while the monitoring and scheduling center is often deployed in the asset management Midwest or comprehensive monitoring system management platform according to the actual situation of rail transit. In the data collection layer, each node will monitor a large amount of raw data, which is a no small task for both the node processor and the data transmission between each intelligent node due to the large scale and poor accuracy. And for some occasions, often only the results of monitoring events are concerned, not the data itself. Therefore, in this part, data fusion techniques need to be designed. Data fusion techniques can be combined in the data acquisition layer, which reduces the amount of data transmission and saves energy overhead, or in the gateway and application layers.

In the communication mode, when the CC2425 sends data, it first writes the data into the sending buffer and starts data transmission when the wireless channel is free. When receiving data, the same data is first stored in the receive buffer, and the status of the received data is output through 
TABle 1: Composition of QR code encoding.

\begin{tabular}{lccc}
\hline Serial number & Constitute & Function & Occupied digits \\
\hline 1 & UUID & Describe the unique identifier of the QR code & Top 16 \\
2 & Information to be encrypted & Store QR code transmission information & Available digits (total capacity-16) \\
\hline
\end{tabular}

the FEFO and FEFOP ports. The flow chart of initialization, sending, and receiving data of the communication module is shown in Figure 1. MF RC522 supports all layers of ISO14443A, and the transmission speed is up to $424 \mathrm{kbps}$. The internal transmitter part can directly drive the shortdistance antenna without adding an active circuit. The receiving part provides solid and effective demodulation and decoding circuit for receive ISO14443A compatible response signal.

2.2. Intelligent Model Construction Based on WSN and RFID Technology. In this paper, the three-dimensionally arranged beacon nodes are projected on a horizontal plane, and an improved localization algorithm is proposed by using the traditional RSSI trilateral measurement algorithm and combining the fusion technology of WSN and RFID. The positioning of blind areas and incomplete coverage of RF signals ineffectively compensated, and the positioning accuracy of RF coverage and motion trajectory is improved.

The format of the localization vector in the localization algorithm is designed as (tag, node ID, $X_{\text {first }}, X_{\text {last }}$ ), where tag denotes the electronic tag carried by the miner and has a unique EPC code, node ID denotes the ID number of the beacon node, tatar denotes the time when the asset is first monitored within the range of the beacon node, and tend denotes the time when the asset is last monitored by the beacon. The time when the asset was last monitored by the beacon node, and the positioning vector after group ordering is shown in Table 2.

Assuming that the current position of the unknown node is not within the range of any beacon node signal, let the unknown node pass through beacon nodes $x_{1}, x_{2}$, and $x_{3}$ in turn with signal radii $d\left(x_{1}\right), d\left(x_{2}\right)$, and $d\left(x_{3}\right)$, and the unknown node is $d\left(k, x_{1}\right)$ at the signal range boundary to $x_{1}, d\left(k, x_{2}\right)$ at the signal range boundary to $x_{2}$, and $d\left(k, x_{3}\right)$ at the signal range boundary to $x_{3}$. The moment to be located is known to be $T_{x}$ and the unknown node. The last monitored moment Tend at $x_{1}$ is $T\left(x_{1}\right)$, as in Equation (1). The distance from the unknown node to the beacon node N1 can be obtained from Equation (1).

$$
d\left(k, x_{1}\right)=f(v, d) *\left(T\left(x_{n}\right)-T\left(x_{1}\right) .\right.
$$

The last monitored moment Tend of the unknown node at $x_{2}$ is $T\left(x_{2}\right)$, as in Equation (2). From Equation (2), the distance from the unknown node to the beacon node is $x_{2}$.

$$
d\left(k, x_{2}\right)=f(v, d) *\left(T\left(x_{n}\right)-T\left(x_{2}\right) .\right.
$$

The moment $X_{\text {first }}$ when entering $x_{3}$ is monitored for the first time that is $T\left(x_{3}\right)$, as in Equation (3). From Equation (3), the distance from the unknown node to $x_{3}$ is obtained.

$$
d\left(k, x_{3}\right)=f(v, d) *\left(T\left(x_{n}\right)-T\left(x_{3}\right) .\right.
$$

Based on the above three assumptions, then the traditional trilateral measurement algorithm is improved to Equation (4).

$$
\left\{\begin{array}{l}
(d(i)+d(k, i))^{2}=\left(f(x)-f\left(x_{i}\right)\right)^{2}+\left(f(y)-f\left(y_{i}\right)\right)^{2} \\
G(m)=\sum_{j=1}^{N}\left(T\left(x_{j}\right)-T(m)\right)^{2}
\end{array}, i=1,2,3,\right.
$$

where the unknown node is within the range of a smart node $x_{i}$, and then $d(k, i)=0$. The joint cubic equation system can calculate the coordinates $(x, y)$ of the unknown node, as shown in Equation (5).

$$
\left\{\begin{array}{l}
x_{1}^{3}-x_{2}^{3}+y_{1}^{3}-y_{2}^{3}=\left(d\left(x_{1}\right)+d\left(k, x_{1}\right)\right)^{3}-\left(d\left(x_{2}\right)+d\left(k, x_{2}\right)\right)^{3}, \\
x_{1}^{3}-x_{3}^{3}+y_{1}^{3}-y_{3}^{3}=\left(d\left(x_{1}\right)+d\left(k, x_{1}\right)\right)^{3}-\left(d\left(x_{3}\right)+d\left(k, x_{3}\right)\right)^{3}, \\
x_{2}^{3}-x_{3}^{3}+y_{2}^{3}-y_{3}^{3}=\left(d\left(x_{2}\right)+d\left(k, x_{2}\right)\right)^{3}-\left(d\left(x_{3}\right)+d\left(k, x_{3}\right)\right)^{3} .
\end{array}\right.
$$

The service life of the sensing tag can be calculated by Equation (6), where $D$ is the battery capacity, $M$ is the duty cycle, and the MCU indicates the state " 0 " when it is working and " 1 " when it is sleeping, and the probability of " 0 " and " 1 " is the same as 0.5 . The duty cycle is $M=0.5$.

$$
P(h)=\frac{D}{\left(L_{\text {active }} * M\right)+L_{\text {sleep }} *(1-M)} .
$$

The carrier frequency is set to grow from $810 \mathrm{MHz}$ to $950 \mathrm{MHz}$ in $5 \mathrm{MHZ}$ steps. The reader antenna is placed at a distance of $d=1 \mathrm{~m}$ from the sensing tag antenna $(H$ (reader $)=6.5 \mathrm{dBi})$, and the reader transmits at a power of $P($ on $)$. The sensitivity of the sensing tag can be obtained by Equation (7), where $P$ (on) denotes the minimum power that can activate the RFID tag. $H$ (ide) takes the maximum antenna gain of the sensing tag as $1.8 \mathrm{dBi}, H$ (ide) $=1.8 \mathrm{dBi}, \alpha$ is the wavelength, and $\beta$ is the polarization loss factor of the reader antenna and the sensing tag antenna.

$$
\left\{\begin{array}{l}
L(\text { ide })=\beta * Q(\text { on }) * H(\text { ide }) *\left(\frac{\lambda * \alpha}{3 \pi d(k, i)}\right)^{2}, \\
Q(\text { on })=P(\text { reader }) * H(\text { reader }) .
\end{array}\right.
$$




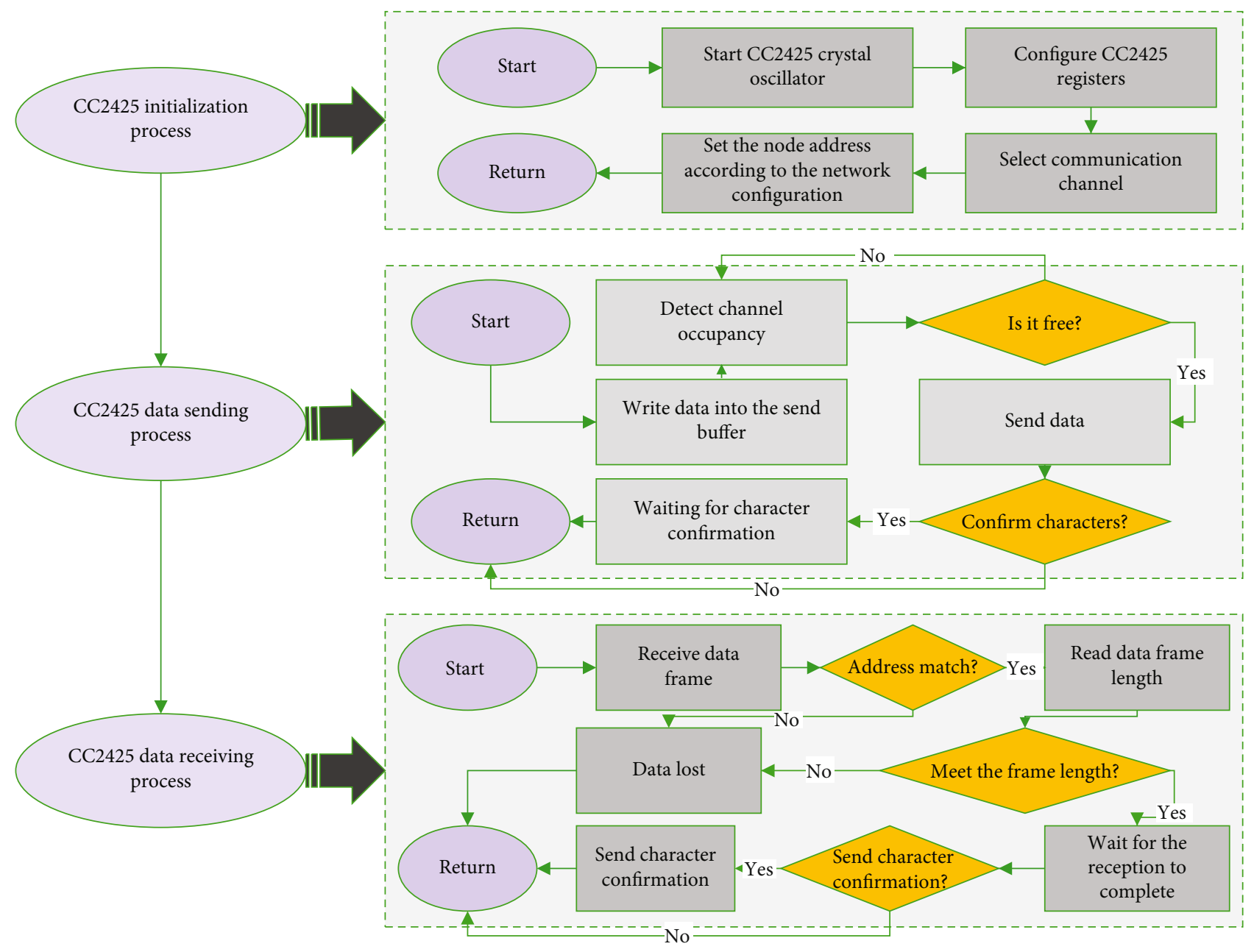

Figure 1: CC2425 program flow chart.

TABle 2: Table of positioning vectors.

\begin{tabular}{lcccc}
\hline Serial number & Tag & Node ID & $X_{\text {first }}$ & $X_{\text {last }}$ \\
\hline 1 & Tag 1 & Node 1 & First 1 & Last 1 \\
2 & Tag 2 & Node 2 & First 2 & Last 2 \\
$\ldots$ & $\ldots$ & $\ldots$ & $\ldots$ & $\ldots$ \\
4 & Tag $N$ & Node $N$ & First $N$ & Last $N$ \\
\hline
\end{tabular}

The read/write distance of UHF RFID tags is an important index for testing the performance of the tags. The maximum read/write distance of the sensing tag is calculated by Equation (8). Evaluate the position of the mobile terminal equipment entering the positioning area and determine the range of the area in which the pending node can exist, and when the RFID reader recognizes the RFID tag of the entered RF area, the reader's data will be related to itself.

$$
\max (d)=\alpha * \frac{\lambda}{3 \pi d} \sqrt{\frac{\max (Q(\text { on })) * H(\text { ide }) * \beta}{U(\text { ide })}} .
$$

The main basis for switching between RFID and ZigBee technologies is the advantages and disadvantages of the communication conditions of the two technologies, and a communication condition detection loop is proposed. The communication condition detection loop consists of two detection tasks for detecting the real-time communication conditions between RFID and ZigBee, and the two detection tasks are defined as RFID_test $\operatorname{loop}()$ and ZigBee_test_loop(). Firstly, RFID_test_loop() is used to detect the RFID communication conditions, if the RFID communication environment is good, the status monitoring IED and the main IED use RFID technology to communicate, if RFID test task RFID_test_loop0 detects that RFID technology cannot meet the communication requirements, then ZigBee technology is used. If the status monitoring IED and the main IED cannot communicate even if they try to use ZigBee technology, then the communication between them fails and the next test task loop will be carried out.

2.3. Intelligent Asset Management System Design and Implementation. The asset management system realizes the 


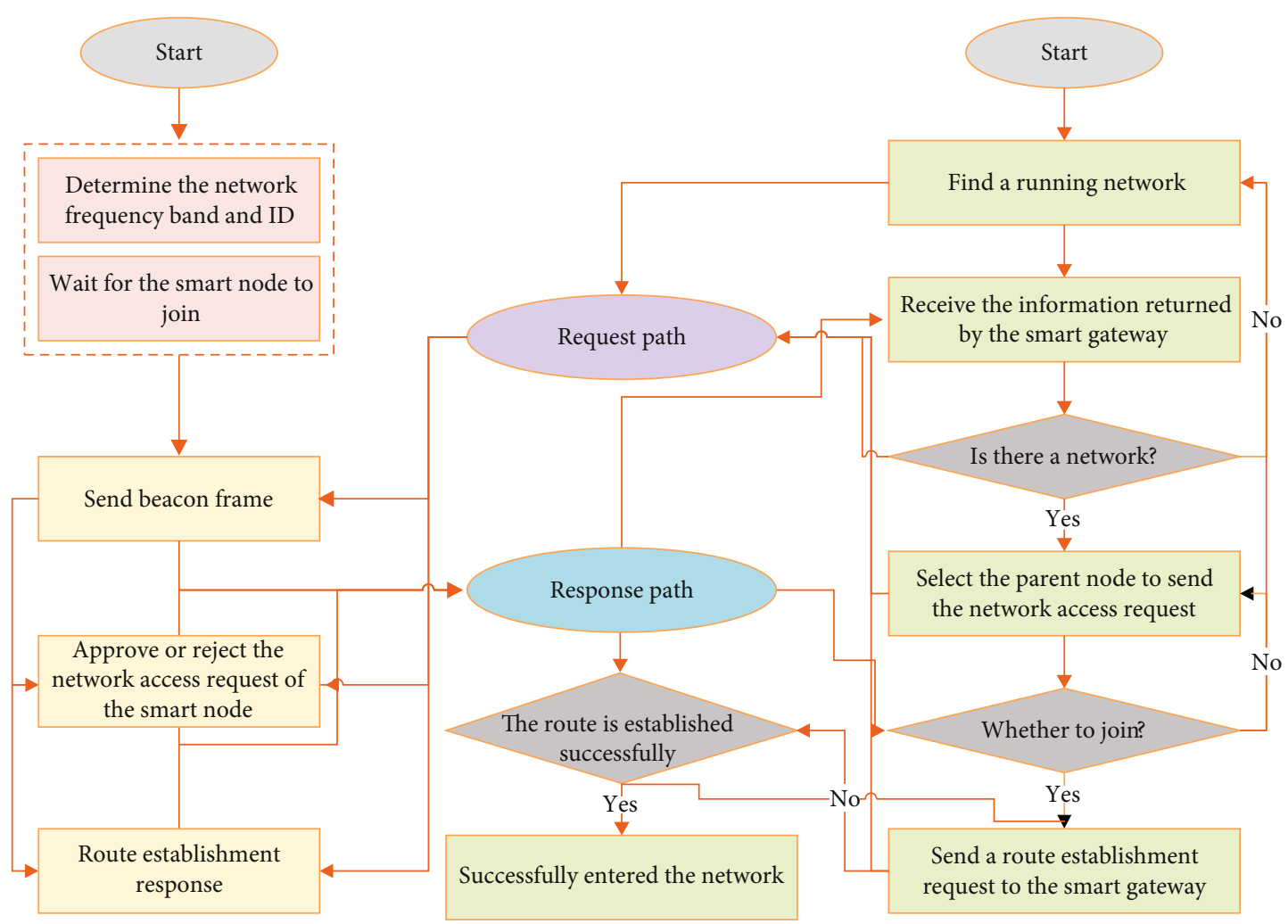

FIgURE 2: Flow chart of network formation.

modules of business management, information inquiry and analysis, system setting, and cell phone application. Before running the system, an initial setup is performed, and some necessary basic information is entered, after which the system can run normally $[21,22]$. To use the system, the identity of the user has to be determined by logging in, and the functional interaction interface is given according to the user's authority. After logging in, you enter the operation interface of the asset management system, which includes the system setting module, business processing module, and system message management module.

The ZigBee coordinator of the smart gateway is responsible for the information collection of the whole data collection layer. Therefore, it needs to be responsible for the networking of smart nodes with the gateway. After successful networking, each node is then responsible for its task under the management of the operating system. The ZigBee protocol has a clear process for the networking process of the system. First, the coordinator module scans and selects all available $2.4 \mathrm{GHz}$ bands in the protocol stack configuration. Then, the band with less noise is selected to build the network, or this step is skipped if there is a present. The coordinator assigns a network address packet of limited capacity to each smart node that is to join the network. The flow chart of the network formation procedure of the smart gateway and the network entry procedure of the smart nodes are shown in Figure 2.

The uppermost layer in the traceability system is the application layer, which is responsible for the management, storage, and display of data. The database is responsible for data collection and storage, and the webserver is responsible for business logic processing and data presentation. The system is designed in $\mathrm{B} / \mathrm{S}$ mode with MySQL database, Nginx web server, Python+Django framework as the development language, and Bootstrap+Jquery as the front-end development, and the design of the application layer mainly include coding design, database design, and website logic design.

The data transmission module is mainly responsible for collecting, organizing, and uploading data. This module is mainly composed of sensing nodes that upload the collected data to the database through the data transmission module. For systems with a large number of data collection nodes, it is appropriate to use a wireless sensor network as the medium for data transmission and collection. Wireless sensor networks are highly integrated and involve a variety of cutting-edge science and technology. The network itself consists of multiple stationery or mobile sensing nodes that collaborate to collect and process data information. Since they transmit simple data, they require low bandwidth, but low latency and power consumption. Very low power consumption can greatly extend the working time of nodes. And ZigBee has the advantages of low complexity, low power consumption, low rate, and low cost; so, this paper uses ZigBee wireless sensor network as a medium for data transmission. ZigBee wireless sensor network has three main logical device types: coordinator (coordinator) router (router), and end-device. Usually, a ZigBee network consists of a coordinator as well as multiple routers and end devices. The functions of each part in the ZigBee network composition are shown in Table 3. 
TABle 3: Functions of each node in the ZigBee network.

\begin{tabular}{|c|c|c|c|}
\hline Serial number & Equipment name & Hardware name & Function \\
\hline \multirow[b]{2}{*}{1} & \multirow{2}{*}{ Coordinator } & CC2425 core board & Network configuration \\
\hline & & CC2425 base plate & Start-up and maintenance \\
\hline \multirow{2}{*}{2} & \multirow{2}{*}{ Router } & CC2425 core board & Node verification \\
\hline & & CC2425 base plate & Data forwarding \\
\hline \multirow{3}{*}{3} & \multirow{3}{*}{ Terminal node } & CC2425 core board & Data upload \\
\hline & & CC2425 base plate & Data forwarding \\
\hline & & Multiple sensors & Data collection \\
\hline
\end{tabular}

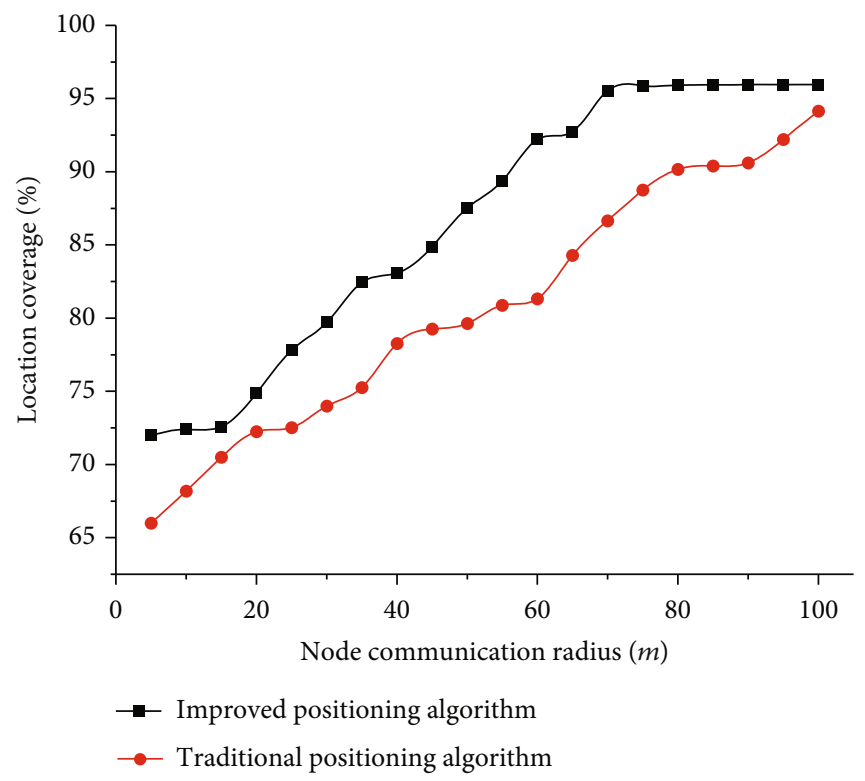

FIgURE 3: Positioning coverage map.

\section{Analysis of Results}

3.1. Intelligent Model Analysis. Comparing the improved localization algorithm in this paper with the traditional localization algorithm, the coverage of the improved localization algorithm approximates 95\% when the localization radius of the beacon nodes is gradually increased. In the traditional localization algorithm, the coverage of localization is only about $70 \%$ when the radius of beacon node communication is short because the beacon node arrangement has a great influence on the localization accuracy, but as the radius of beacon node communication increases, the coverage of the traditional algorithm and the improved algorithm will converge. From Figure 3, it can be shown that the coverage rate of the improved algorithm can be significantly higher than that of the traditional algorithm.

In the experiment, the communication radius is set to $10 \mathrm{M}$ in this paper, and the Markov chain is effectively used for localization trajectory prediction, using the localization coordinates of a known moment to predict the localization coordinates of the next unknown moment, in turn. The experiment will be run using 50 times of the same data and take its average. The localization error of one of the experiments is shown in Figure 4(a). Using the same method, the average error of the predicted 50, 70, and 100 motion trajectories is compared, as shown in Figure 4(b). From Figure 4, it can be obtained that the $X$-axis and $Y$ -axis coordinates of the localization coordinates are stable in the range of 100 meters, and the error values are more stable. However, in the subsequent time slot prediction, the error value gradually increases. It is found that it is due to the accumulation error of the Markov chain itself. In practical applications, the combination of the positioning algorithm and the prediction algorithm in this paper can effectively reduce the prediction error.

According to Figure 5, it can be seen that the improved algorithm in this paper significantly reduces the localization error when there are fewer nodes compared with the traditional algorithm. This is because when the reference nodes are relatively few, the influence coefficient of environmental errors on system localization is larger, and the RSSI filtering model, as well as the location filtering in this paper, just reduces the influence of environmental factors on localization. As the number of reference nodes increases, both the 


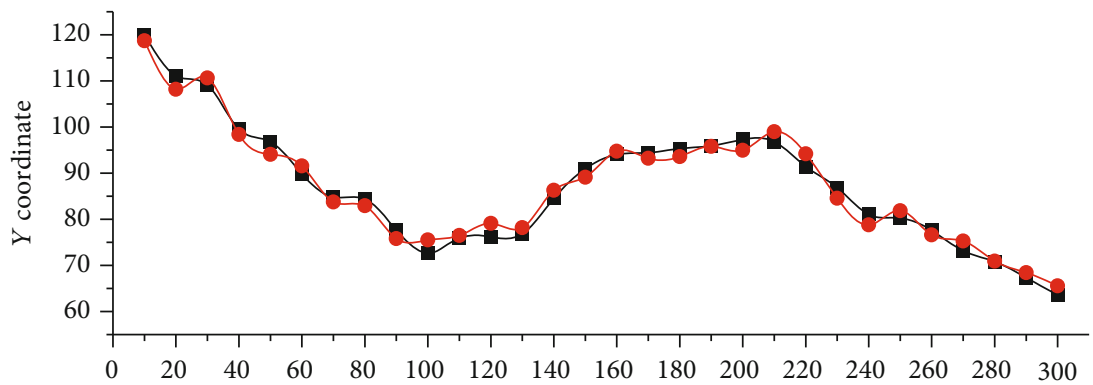

(a)

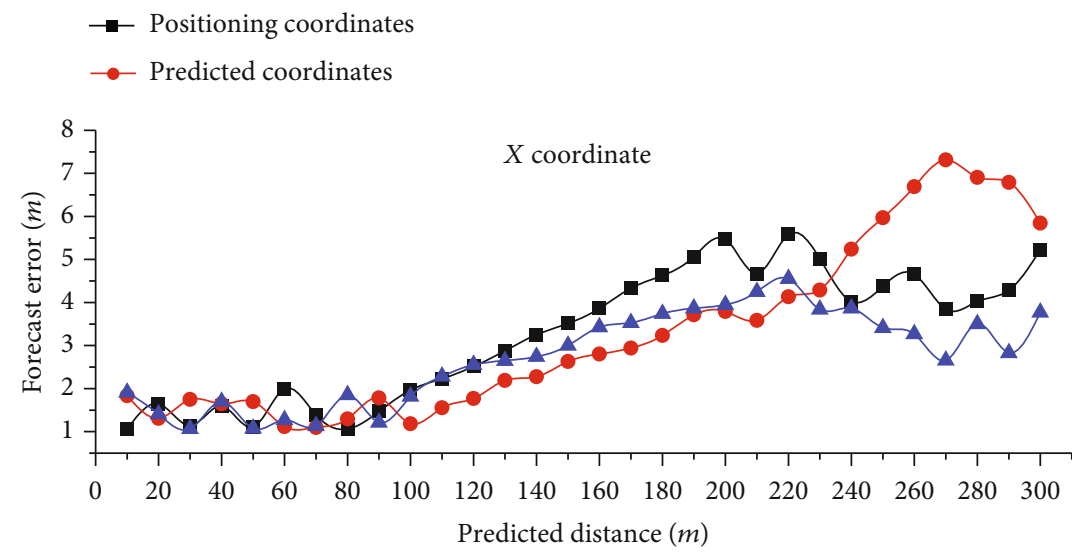

(b)

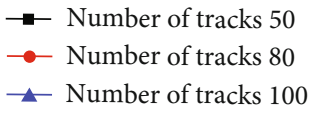

FIGURE 4: Comparison of predicted position with localized position and overall predicted localization error analysis.

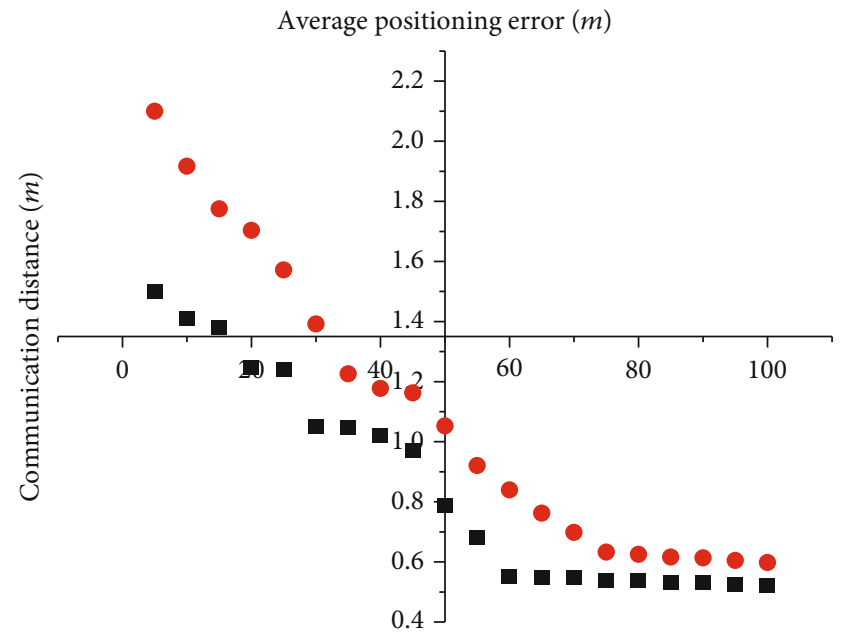

- Improved positioning algorithm

- Traditional positioning algorithm

FIgURE 5: Average positioning error.

improved algorithm and the traditional algorithm improve inaccuracy. The reason for this phenomenon is that the algorithm takes the geometric mean at the end so that the environmental error has less influence on the final localization results.
3.2. Analysis of the Intelligent Asset Management System. Simulation experiments of wireless sensor networks use 5hop binary tree static topology. In practical applications, the topology of wireless sensor networks is often irregular and changing. In this paper, a typical 5-hop binary tree static topology is used to study the energy consumption of the RFID RF wake-up mechanism, IEEE802.16.4 MAC protocol, and C-MAC protocol. The energy consumption of the RFID RF wake-up mechanism, IEEE802.16.4 MAC protocol, and C-MAC protocol when $S$ is set to $50 \mathrm{~s}$ is shown in Figure 6. From Figure 6, the energy consumption of the RFID RF wake-up mechanism is the lowest, and the energy consumption of the three protocols is independent of the number of hops. The minimum power consumption of the RFID radio frequency wake-up mechanism reaches 0.53 , and the maximum power consumption of the IEEE802.15.4 MAC protocol reaches 2.71 .

Figure 7 shows the simulation and test results of the system antenna return loss; as shown in Figure 7, the test results are very close to the simulation results. The test result shows that the antenna center frequency is $600 \mathrm{MHz}$, and $\mathrm{S} 11 \mathrm{~min}$ imum is about $-14.91 \mathrm{~dB}$. The simulation result shows that the antenna center frequency is $900 \mathrm{MHz}$, and $\mathrm{S} 11$ minimum is about $-14.54 \mathrm{~dB}$. The rationality of RFID tag antenna design depends on, whether the antenna impedance and chip impedance to achieve a perfect match, the antenna impedance, and chip impedance to achieve matching can 

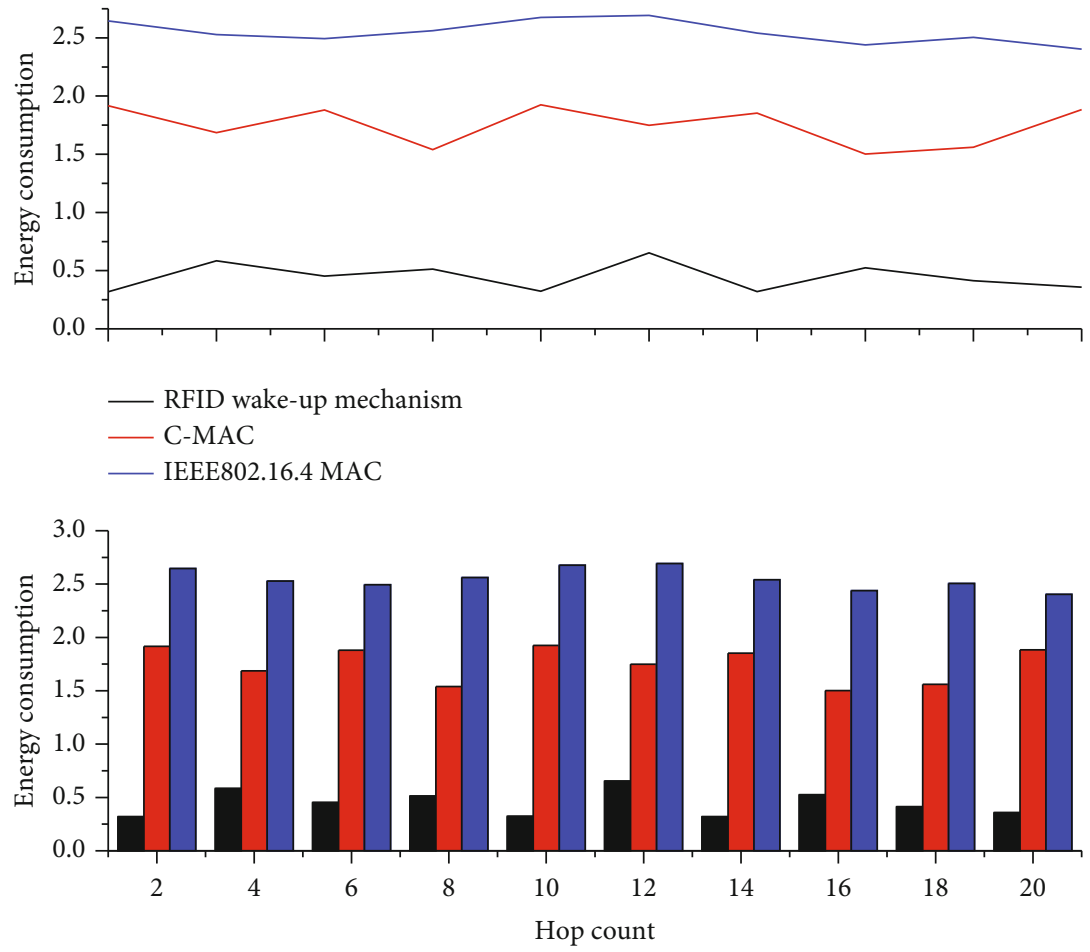

RFID wake-up mechanism

C-MAC

IEEE802.16.4 MAC

Figure 6: Comparison of energy consumption of the 3 protocols.

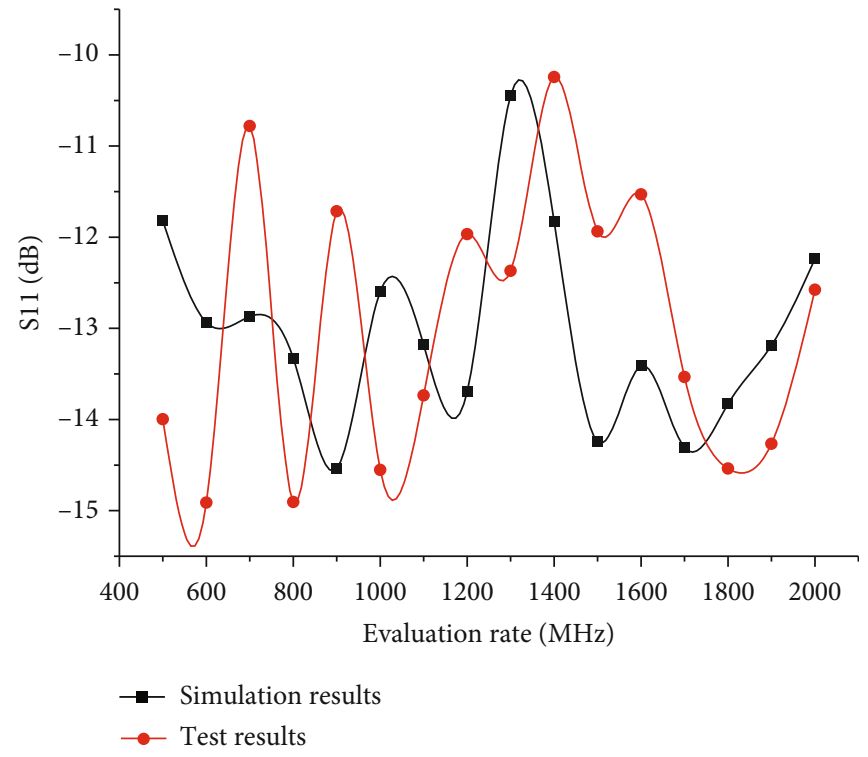

Figure 7: System antenna return loss.

improve the power supply efficiency and read-write distance of the tag antenna. The input impedance of the antenna should be designed as the common impedance of the tag chip, to achieve the maximum energy transfer.

In the performance testing of the fixed asset equipment system, since the system may be used by multiple users at the same time, multiple users are simulated to conduct random access operations to each server of the system at the same time, the corresponding processing time of each server operation is recorded, and the performance of the test results is evaluated and compared. The designed test scenario is as follows: the database is initially entered with 

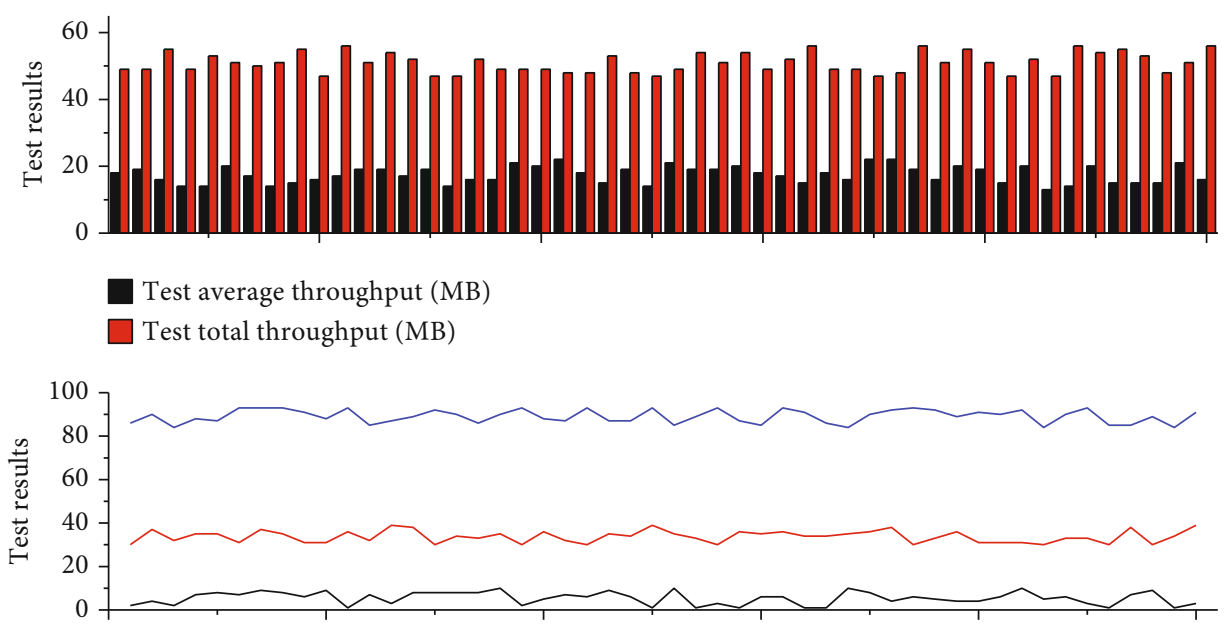

_ Minimum event processing time (ms)

_ Average event processing time (ms)

- Maximum event processing time (ms)

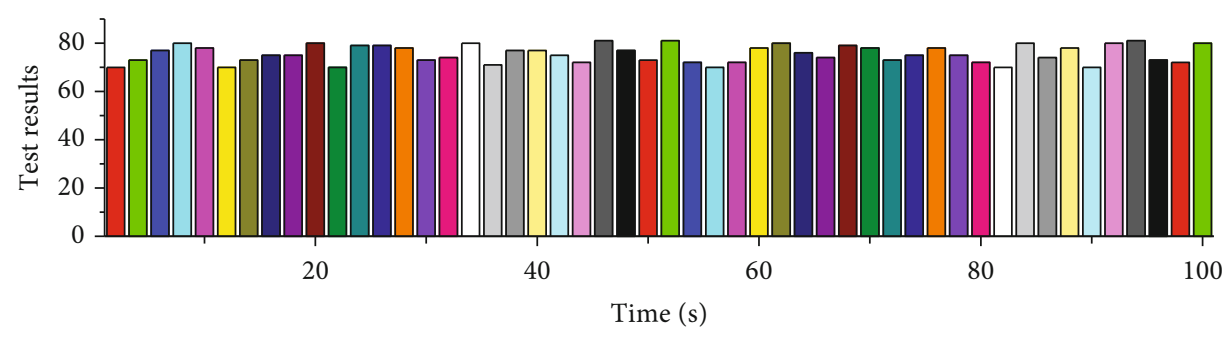

Total number of incident processing (1000 items)

Figure 8: Asset system performance test chart.

more than 10000 basic data, and more than 200 users with different roles are simulated to access the fixed asset equipment management system concurrently and randomly, and the test access time is tested by random access to these users (Figure 8).

\section{Conclusion}

Based on the discussion of RFID technology and WSN technology, this paper finds that RFID technology and WSN technology are two functional complementary technologies and therefore consider the fusion of RFID technology and WSN technology. An intelligent asset management system architecture based on RFID and WSN fusion technology is proposed, which is divided into information sensing layer, data communication layer, information fusion layer, and integrated application layer, and the functions realized by each layer are introduced in detail. The system in this paper has been analyzed and designed to complete the development work, and in this design implementation process, the development status and background of the asset system are introduced. The corresponding software development techniques are applied. It also follows the software program design concepts, such as the system can run permanently, the system security performance is reliable, the user is easy to operate, the system has strong scalability, and other pro- gram development design theories. The main functional modules are implemented by analyzing the requirements of the asset system. For example, the system management, asset management, asset query, and report management modules finally realize the whole system from front-end UI to backend logic code writing. After the system development was completed, the functional and performance tests of the fixed asset management system were conducted according to the common testing methods and theoretical principles of the system. The results fully demonstrate that the developed asset system is safe and reliable and can ensure the efficient management of assets. For the intelligent asset management network proposed in this paper, a comprehensive and detailed analysis is not carried out, and the hardware design involved is only the introduction of the design method, which is not relevant. In the design of an intelligent asset management system, a reasonable hardware scheme should be adopted according to the demand. Especially sensor module, $\mathrm{RF}$ read/write module, the choice of different locations has great variability.

\section{Data Availability}

The data used to support the findings of this study are available from the corresponding author upon request. 


\section{Conflicts of Interest}

The authors declare that they have no known competing financial interests or personal relationships that could have appeared to influence the work reported in this paper.

\section{Acknowledgments}

This study was supported by the National Social Science Foundation of China: research on the coordination of monetary policy and macro prudential policy from the perspective of operational tools (13BJY165).

\section{References}

[1] F. Wan and M. Li, "RFID based intelligent traffic information acquisition system," International Journal of RF Technologies, vol. 11, no. 1, pp. 45-58, 2020.

[2] X. Gaofeng, "Low financial cost with ant colony optimisation in intelligent agriculture," International Journal of Wireless and Mobile Computing, vol. 18, no. 2, pp. 111-115, 2020.

[3] A. Dawson, "Robotic wireless sensor networks, big data-driven decision-making processes, and cyber-physical system-based real-time monitoring in sustainable product lifecycle management," Economics, Management, and Financial Markets, vol. 16, no. 2, pp. 95-105, 2021.

[4] R. Granillo-Macías, I. Simón-Marmolejo, I. J. GonzálezHernández, and J. Zuno-Silva, "Traceability in Industry 4.0: A Case Study in the Metalmechanical Sector," Acta Logística, vol. 7, no. 2, pp. 95-101, 2020.

[5] F. Molaei, E. Rahimi, H. Siavoshi, S. G. Afrouz, and V. A. Tenorio, "A comprehensive review on internet of things (IoT) and its implications in the mining industry," American Journal of Engineering and Applied Sciences, vol. 13, no. 3, pp. 499-515, 2020.

[6] K. Leng, L. Jin, W. Shi, and I. van Nieuwenhuyse, "Research on agricultural products supply chain inspection system based on internet of things," Cluster Computing, vol. 22, Supplement 4, pp. 8919-8927, 2019.

[7] P. Gibson and J. Macek, "Sustainable industrial big data, automated production processes, and smart networked factories in cyber-physical system-based manufacturing," Economics, vol. 9, no. 3, pp. 22-34, 2021.

[8] M. A. M. Sadeeq and S. Zeebaree, "Energy management for internet of things via distributed systems," Journal of Applied Science and Technology Trends, vol. 2, no. 2, pp. 59-71, 2021.

[9] E. Cunningham, "Artificial intelligence-based decisionmaking algorithms, sustainable organizational performance, and automated production systems in big data-driven smart urban economy," Economics, vol. 6, no. 1, pp. 31-41, 2021.

[10] S. K. Kinnunen, A. Ylä-Kujala, S. Marttonen-Arola, T. Kärri, and D. Baglee, "Internet of things in asset management," Management, Engineering, and Technology (IJSSMET), vol. 9, no. 2, pp. 104-119, 2018.

[11] M. Andreacchio, A. Bekrar, R. Benmansour, and D. Trentesaux, "Assessing cyber-physical systems to balance maintenance replacement policies and optimise long-run average costs for aircraft assets," IET Cyber-Physical Systems: Theory \& Applications, vol. 4, no. 2, pp. 148-155, 2019.

[12] C. Çeken and D. Abdurahman, "Simulation modeling of an iot based cold chain logistics management system," Sakarya Uni- versity Journal of Computer and Information Sciences, vol. 2, no. 2, pp. 89-100, 2019.

[13] L. Qin, S. Feng, and H. Zhu, "Research on the technological architectural design of geological hazard monitoring and rescue-after-disaster system based on cloud computing and internet of things," International Journal of System Assurance Engineering and Management, vol. 9, no. 3, pp. 684-695, 2018.

[14] J. I. Z. Chen and K. L. Lai, "Machine learning based energy management at Internet of Things network nodes," Journal: Journal of Trends in Computer Science and Smart Technology September, vol. 2020, no. 3, pp. 127-133, 2020.

[15] J. Chen, "Composition rule perception algorithm of national art plane system based on wireless sensor network communication technology," International Journal of Wireless Information Networks, vol. 28, no. 3, pp. 243-251, 2021.

[16] C. K. Lee, Y. Lv, K. K. Ng, W. Ho, and K. L. Choy, "Design and application of internet of things-based warehouse management system for smart logistics," International Journal of Production Research, vol. 56, no. 8, pp. 2753-2768, 2018.

[17] W. Zhang, M. Kumar, and J. Liu, "Multi-parameter online measurement IoT system based on BP neural network algorithm," Neural Computing and Applications, vol. 31, no. 12, pp. 8147-8155, 2019.

[18] M. Ben-Daya, E. Hassini, and Z. Bahroun, "Internet of things and supply chain management: a literature review," International Journal of Production Research, vol. 57, no. 15-16, pp. 4719-4742, 2019.

[19] A. I. Vlasov, P. V. Grigoriev, A. I. Krivoshein, V. A. Shakhnov, S. S. Filin, and V. S. Migalin, "Smart management of technologies: predictive maintenance of industrial equipment using wireless sensor networks," Entrepreneurship and Sustainability Issues, vol. 6, no. 2, pp. 489-502, 2018.

[20] B. S. Awoyemi, A. S. Alfa, and B. T. J. Maharaj, "Resource optimisation in 5G and internet-of-things networking," Wireless Personal Communications, vol. 111, no. 4, pp. 2671-2702, 2020.

[21] B. Bhushan, C. Sahoo, P. Sinha, and A. Khamparia, "Unification of Blockchain and Internet of Things (BIoT): requirements, working model, challenges and future directions," Wireless Networks, vol. 27, no. 1, pp. 55-90, 2021.

[22] Y. Ding, M. Jin, S. Li, and D. Feng, "Smart logistics based on the internet of things technology: an overview," International Journal of Logistics Research and Applications, vol. 24, no. 4, pp. 323-345, 2021. 